\title{
Ambient Spaces of Dimensional Dual Arcs
}

\author{
SATOSHI YOSHIARA* \\ yoshiara@cc.osaka-kyoiku.ac.jp \\ Division of Mathematical Sciences, Osaka Kyoiku University, Kashiwara, Osaka, 582-8582, Japan
}

Received August 8, 2002; Revised February 11, 2003; Accepted February 27, 2003

\begin{abstract}
A $d$-dimensional dual arc in $P G(n, q)$ is a higher dimensional analogue of a dual arc in a projective plane. For every prime power $q$ other than 2 , the existence of a $d$-dimensional dual arc $(d \geq 2)$ in $P G(n, q)$ of a certain size implies $n \leq d(d+3) / 2$ (Theorem 1). This is best possible, because of the recent construction of $d$-dimensional dual arcs in $P G(d(d+3) / 2, q)$ of size $\sum_{i=0}^{d-1} q^{i}$, using the Veronesean, observed first by Thas and van Maldeghem (Proposition 7). Another construction using caps is given as well (Proposition 10).
\end{abstract}

Keywords: dual arc, dual hyperoval, Veronesean

\section{Introduction}

Let $q=p^{e}$ be a power of a prime $p$ with $e \geq 1$, and let $G F(q)$ be the field of $q$ elements. To distinguish the dimension as a projective space and that as a vector space, we use the terminology rank to refer to the latter. For a vector space $V$ of rank $n+1$ over $G F(q)$, $P G(V)$ denotes the associated Desarguesian projective space of dimension $n$. We denote by $P G(n, q)$ the isomorphism class of $P G(V)$. Sometimes $P G(V)$ is used to indicate the set of projective points of $P G(V)$. The number of projective points of $P G(n, q)$ is denoted

$$
\theta_{q}(n):=q^{n}+q^{n-1}+\cdots+1=\left(q^{n+1}-1\right) /(q-1),
$$

or simply $\theta(n)$.

A family $\mathcal{A}$ of $d$-dimensional subspaces of $P G(n, q)$ with $n \geq 2$ is called a $d$-dimensional dual arc in $P G(n, q)$, if it satisfies the following three axioms:

(DA1) every two distinct members of $\mathcal{A}$ intersect at a projective point,

$(D A 2)$ every three distinct members of $\mathcal{A}$ intersect trivially, and

(DA3) all members of $\mathcal{A}$ span the whole space $P G(n, q)$.

The space $P G(n, q)$ is called the ambient space of the dual $\operatorname{arc} \mathcal{A}$. The dual arc $\mathcal{A}$ is called complete if there is no $d$-dimensional dual arc containing $\mathcal{A}$ properly.

A $d$-dimensional dual arc $\mathcal{A}$ consists of at most $\theta_{q}(d)+1$ members, because for a member $X$ of $\mathcal{A}$, the map $\mathcal{A} \backslash\{X\} \ni Y \mapsto X \cap Y \in P G(X)$ (the set of projective points

*Present address: Department of Mathematics, Tokyo Woman's Christian University, Suginami-ku, Tokyo, 1678585, Japan. 
of $P G(X) \cong P G(d, q))$ is well-defined and injective by the axioms (DA1) and $(D A 2)$. A $d$-dimensional dual arc $\mathcal{A}$ in $P G(n, q)$ is called a dual hyperoval, if this upper bound is attained: $|\mathcal{A}|=\theta_{q}(d)+1$.

It is not difficult to see that the existence of a 1-dimensional dual arc (of at least three members) in $P G(n, q)$ implies $n=2$, and that the notion of 1-dimensional dual arcs coincides with that of dual arcs in $P G(2, q)$, that is, a set of lines no three of which contains a point in common. Thus the notion of dual hyperovals and arcs can be thought of as a higher dimensional analogue of that of dual hyperovals and dual arcs in a projective plane. Hence, we only consider the case $d \geq 2$, throughout the paper.

The notion of dual hyperovals was first appeared in the paper by Huybrechts and Pasini [6], and that of dual arc was introduced by the author [12, Section 2] as its generalization. See $[7,8]$ for relations of dual hyperovals with semibiplanes and a class of distance regular graphs.

Several fundamental properties of dual hyperovals were obtained by Del Fra [3] as well as the classification of 2-dimensional dual hyperovals. Specifically, he succeeded in characterizing the remarkable 2-dimensional dual hyperoval in $P G(5,4)$ with the automorphism group the Mathieu group $M_{22}$, in a geometric property, the property $(T)$ (Proposition 11). Characterization of $d$-dimensional dual hyperovals in $P G(2 d, q)$ was achieved by Cooperstein and Thas [2].

As for constructions, Huybrechts [5] gave a $d$-dimensional dual hyperoval in $P G(D, 2)$, where $D=d(d+3) / 2$, for every $d$, and the author gave a family of them in $P G(2 d+1,2)$ [11] which is doubly indexed by the numbers $m, n$ with $1 \leq m, n \leq d$ coprime to $d+1$ for every $d$. The former can be thought of as an example of the "cap construction" of dual arcs (Section 3.2). Recently, Thas and van Maldeghem provide several characterizations of the Veronesean [9, 10], the "second" of which [10, Section 3] is based on the following observation: for every prime power $q$ and $d \geq 2$, using the Veronesean, we can construct a $d$-dimensional dual arc of size $\theta_{q}(d)$ in $P G(D, q), D=d(d+3) / 2$. (Note that the properties $(V S i)(i=1,2,3)$ given in [10, Section 3] are the dual form of the axioms ( $D A i)(i=1,2,3)$ above.) It can be extended to a dual hyperoval if and only if $q$ is even (Corollary 8 in Section 3). Remark that for $q=2$ the $d$-dimensional dual hyperoval in $P G(D, 2)$ constructed by Huybrechts is not isomorphic to the dual hyperoval by Thas and van Maldeghem (Proposition 11).

When there exists a $d$-dimensional dual arc in $P G(n, q)$, then we have an obvious lower bound $2 d \leq n$, because $2 d$ is the dimension of the subspace of $\operatorname{P} G(n, q)$ spanned by two members of the dual arc by the axiom ( $D A 1)$. Then it is natural to ask:

Find an upper bound on the dimension $n$ of the ambient space $P G(n, q)$ of a $d$-dimensional (complete) dual arc, in terms of $d$ and $q$.

Del Fra [3, Proposition 2.3] gave the following bound when there exists a $d$-dimensional dual hyperoval in $P G(n, q)$ :

$$
n<2 d+\sum_{i=1}^{d-1} i q^{d-i} .
$$

The main aim of the present paper is to provide an improvement of his bound. 
Theorem 1 Assume that $\mathcal{A}$ is a d-dimensional dual arc in $P G(n, q)$.

(i) If $q>2$ and $|\mathcal{A}| \geq \theta(d)-\alpha(q)$, where

$$
\alpha(q)=\left\{(q-3) q^{d+1}+\left(q^{d}-q^{2}\right)+(q-1) d+(3 q-1)\right\} /(q-1)^{2},
$$

then $n \leq d(d+3) / 2=: D$.

(ii) If $q=2$ and $\mathcal{A}$ is a dual hyperoval, then $n \leq D+2$.

Observe that the number $\alpha(q)$ in the statement (i) above is positive for every prime power $q$ other than 2 and every $d \geq 2$. The upper bound in (i) is specifically valid for dual hyperovals as well as dual arcs of second-maximum size $\theta_{q}(d)$. In view of the construction by Thas and van Maldeghem mentioned above, we conclude that the upper bound for $n$ given in (i) is best possible for every prime power $q$ other than 2 and every $d \geq 2$.

As every $d$-dimensional dual arc in $P G(n, q)$ of size $\theta_{q}(d)$ can be uniquely extended to a dual hyperoval if $q$ is even (Proposition 9), the upper bound in (ii) is valid for a dual arc $\mathcal{A}$ of size $\theta_{2}(d)$ as well. It is likely that $n \leq D$ even in the case $q=2$, though the author has not yet succeeded to show that. When $1 \leq d \leq 5$, we have the bound $n \leq D$ (see Proposition 5).

The proof of theorem will be given in Section 2. In Section 3, several constructions of dual arcs will be given, including expositions for the construction by Thas and van Maldeghem.

\section{Proof of theorem}

Straightforward calculations give the following, which gives an account for the value $\alpha(q)$ in Theorem 1(i).

Lemma 2 We have $\alpha(q)=\theta(d)-\theta(d-1)-\sum_{l=1}^{d-1} \theta(l)-2$.

(Note that the value $\alpha(q)$ is positive if $q \geq 3$, while it is negative when $q=2$.)

We now prepare two lemmas on the intersections of members of a dual arc in $P G(n, q)$ with subspaces of $P G(n, q)$. Note that Lemma 4 below is valid only for a dual hyperoval, and will not be used in the proof of Theorem 1(i).

Lemma 3 Let $\mathcal{A}$ be a d-dimensional dual arc in $P G(n, q)$. Assume that a subspace $W$ of $P G(n, q)$ contains at least $\theta(m)+1$ distinct members of $\mathcal{A}$ for some $0 \leq m \leq d-1$. Then every member $X$ of $\mathcal{A}$ intersects $W$ in a subspace of dimension at least $m+1$ : $\operatorname{dim}(X \cap W) \geq m+1$. In particular, if $m=d-1$, then $W=P G(n, q)$.

Proof: Let $X_{i}(i=0, \ldots, \theta(m))$ be distinct members of $\mathcal{A}$ contained in $W$. Then for every $X \in \mathcal{A}$, either $X=X_{i}$ for some $i=0, \ldots, \theta(m)$ or $X$ contains $\theta(m)+1$ distinct points $X \cap X_{i}(i=0, \ldots, \theta(m))$. In either case, $\operatorname{dim}(X \cap W) \geq m+1$, as no subspace of dimension at most $m$ contains $\theta(m)+1$ distinct points. 
When $m=d-1$, we have $\operatorname{dim}(X \cap W)=\operatorname{dim}(X)=d$. Thus $X \subseteq W$ for every member $X \in \mathcal{A}$, and hence $W=\langle\mathcal{A}\rangle=P G(n, q)$.

Lemma 4 Let $\mathcal{H}$ be a d-dimensional dual hyperoval in $P G(n, q)$. Assume that every member of $\mathcal{H}$ intersects a subspace $W$ of $P G(n, q)$ in a subspace of dimension at least $d-1$. Then $n \leq \operatorname{dim}(W)+1$.

Proof: If $W$ contains all members of $\mathcal{H}$, then $W=\langle\mathcal{H}\rangle=P G(n, q)$ and the lemma follows. Thus we may assume that there exists a member $Y$ of $\mathcal{H}$ with $\operatorname{dim}(Y \cap W)=d-1$. Then there are precisely $\theta(d)-\theta(d-1)=q^{d}$ points in $Y$ outside $Y \cap W$. For such a point $P$, there is a unique member $X(P)$ of $\mathcal{H}$ distinct from $Y$ with $Y \cap X(P)=\{P\}$, as $\mathcal{H}$ is a dual hyperoval. As $\operatorname{dim}(X(P) \cap W) \geq d-1$ by our assumption, we have

$$
X(P)=\langle X(P) \cap W, P\rangle \subset\langle W, Y\rangle .
$$

Thus the subspace $\langle W, Y\rangle$ of $P G(n, q)$ contains at least $q^{d}\left(>\theta(d-1)=\left(q^{d}-1\right) /(q-1)\right)$ distinct members of $\mathcal{H}$, and hence $P G(n, q)=\langle W, Y\rangle$ by Lemma 3. Then $n=\operatorname{dim} W+$ $\operatorname{dim} Y-\operatorname{dim}(W \cap Y)=\operatorname{dim}(W)+1$.

We now begin the proof of Theorem 1 . Let $\mathcal{A}$ be a $d$-dimensional dual arc in $P G(n, q)$ with $n \geq 2$. First, we fix two distinct members $A$ and $B$ of $\mathcal{A}$, and define

$$
\mathcal{R}_{0}:=\mathcal{A}-\{A, B\}, U_{0}:=\langle A, B\rangle, \text { and }
$$

$d-l_{0}:=$ the smallest dimension of the subspaces $U_{0} \cap X$ for $X$ ranging over $\mathcal{R}_{0}$.

If $l_{0}=0$, then $\operatorname{dim}\left(U_{0} \cap X\right)=d$ for every $X \in \mathcal{A}-\{A, B\}$, or equivalently $U_{0}$ contains all members of $\mathcal{A}$, as $\operatorname{dim}(X)=d$ for $X \in \mathcal{A}$. Then $U_{0}$ coincides with $\langle\mathcal{A}\rangle=P G(n, q)$ and $n=\operatorname{dim}\left(U_{0}\right)=\operatorname{dim}(A)+\operatorname{dim}(B)-\operatorname{dim}(A \cap B)=2 d$. Hence we may assume that $l_{0} \geq 1$.

Furthermore, observe that $d-1 \geq l_{0}$ : for, each $X \in \mathcal{R}_{0}$ intersects $U_{0}$ in a subspace of dimension at least $1=d-(d-1)$, as $U_{0} \cap X$ contains the line through two distinct points $A \cap X$ and $B \cap X$; and therefore

$$
d-l_{0}=\min \left\{\operatorname{dim}\left(U_{0} \cap X\right) \mid X \in \mathcal{R}_{0}\right\} \geq d-(d-1)
$$

Next, we inductively define subsets $\mathcal{R}_{i}$ of $\mathcal{A}$, subspaces $U_{i}$ of $P G(n, q)$, and non-negative integers $l_{i}$ as follows, until we reach at the stage when $l_{i}=0$.

Assume that $\mathcal{R}_{i}$ and $U_{i}$ are given. Let $d-l_{i}$ be the smallest dimension of $U_{i} \cap X$ for $X$ ranging over $\mathcal{R}_{i}$. We may assume $l_{i} \geq 1$. Let $X_{i}$ be a member of $\mathcal{R}_{i}$ satisfying $\operatorname{dim}\left(U_{i} \cap X_{i}\right)=d-l_{i}$. Then define

$$
\begin{aligned}
\mathcal{R}_{i+1} & :=\left\{X \in \mathcal{R}_{i}-\left\{X_{i}\right\} \mid X \cap X_{i} \notin U_{i} \cap X_{i}\right\}, \\
U_{i+1} & :=\left\langle U_{i}, X_{i}\right\rangle, \text { and } \\
d-l_{i+1} & :=\min \left\{\operatorname{dim}\left(U_{i+1} \cap X\right) \mid X \in \mathcal{R}_{i+1}\right\} .
\end{aligned}
$$


Remark that $\operatorname{dim}\left(U_{i+1} \cap X\right) \geq d-l_{i}+1$ for every $X \in \mathcal{R}_{i+1}$ : for, $X$ contains the subspace $X \cap U_{i}$ of dimension at least $d-l_{i}$ (as $X \in \mathcal{R}_{i+1} \subset \mathcal{R}_{i}$ ) as well as a point $X \cap X_{i}$ which is outside $X \cap U_{i}$ by the definition of $\mathcal{R}_{i+1}$. Thus $l_{i}>l_{i+1}$.

Hence we have a properly decreasing sequence of non-negative integers

$$
d>l_{0}>l_{1}>\ldots,
$$

and therefore there exists a positive integer $k$ such that

$$
1 \leq k \leq d-1, l_{k-1} \geq 1 \text { but } l_{k}=0
$$

(Note that $l_{0} \geq 1$.)

By the definition of $l_{i}$, the condition $l_{k}=0$ implies that $d=\operatorname{dim}\left(U_{k} \cap X\right)$ for every $X \in$ $\mathcal{R}_{k}$, and hence the subspace $U_{k}$ contains all members of $\mathcal{R}_{k}$ (and $\mathcal{R}_{k+1}=\emptyset$ ). Conversely, the condition $\mathcal{R}_{i+1}=\varnothing$ implies that $l_{i}=0$.

For every $i=1, \ldots, k$, the subspace $U_{i}$ also contains $2+i$ members $A, B, X_{0}, \ldots, X_{i-1}$, none of which is a member of $\mathcal{R}_{i}$. This remark with the above observation implies that the subspace $U_{k}$ contains at least $\left|\mathcal{R}_{k}\right|+k+2$ distinct members of $\mathcal{A}$.

Furthermore, we inductively have

$$
\begin{aligned}
\operatorname{dim}\left(U_{i}\right) & =\operatorname{dim}\left(U_{i-1}\right)+\operatorname{dim}\left(X_{i-1}\right)-\operatorname{dim}\left(U_{i-1} \cap X_{i-1}\right) \\
& =\operatorname{dim}\left(U_{i-1}\right)+l_{i-1}=\cdots \\
& =\operatorname{dim}\left(U_{0}\right)+l_{0}+\cdots+l_{i-1}=2 d+\sum_{j=0}^{i-1} l_{j},
\end{aligned}
$$

for $i=1, \ldots, k$.

We will now find a lower bound for the size of $\mathcal{R}_{i}$ for $1 \leq i \leq k-1$ (so that $l_{i} \geq 1$ ). The map sending each member $X$ of $\mathcal{R}_{i}-\left\{X_{i}\right\}$ to the point $X \cap X_{i}$ is an injective map from $\mathcal{R}_{i}-\left\{X_{i}\right\}$ into the set of points of $X_{i}$. As no three distinct members of $\mathcal{A}$ contain a common point, no member of $\mathcal{R}_{i}-\left\{X_{i}\right\}$ intersects $X_{i}$ at one of the following $i+2$ points:

$$
A \cap X_{i}, B \cap X_{i}, X_{0} \cap X_{i}, \ldots, X_{i-1} \cap X_{i}
$$

Thus there are at most $\theta\left(d-l_{i}\right)-(i+2)$ members of $\mathcal{R}_{i}-\left\{X_{i}\right\}$ intersecting $X_{i}$ at some points of the $\left(d-l_{i}\right)$-dimensional subspace $U_{i} \cap X_{i}$ of $X_{i}$. As $\mathcal{R}_{i+1}$ consists of the members of $\mathcal{R}_{i}-\left\{X_{i}\right\}$ intersecting $X_{i}$ at points outside $U_{i} \cap X_{i}$, we have

$$
\begin{aligned}
\left|\mathcal{R}_{i+1}\right| & \geq\left(\left|\mathcal{R}_{i}\right|-1\right)-\left\{\theta\left(d-l_{i}\right)-(i+2)\right\} \\
& =\left|\mathcal{R}_{i}\right|-\theta\left(d-l_{i}\right)+i+1 .
\end{aligned}
$$

Therefore, we inductively have the following estimate:

(*) $\left|\mathcal{R}_{i}\right| \geq\left|\mathcal{R}_{i-1}\right|-\theta\left(d-l_{i-1}\right)+i \geq \cdots$ 


$$
\begin{aligned}
& \geq\left|\mathcal{R}_{0}\right|-\sum_{j=0}^{i-1} \theta\left(d-l_{j}\right)+\sum_{m=1}^{i} m \\
& =(|\mathcal{A}|-2)-\sum_{j=0}^{i-1} \theta\left(d-l_{j}\right)+i(i+1) / 2 .
\end{aligned}
$$

We now separate the cases according as $q>2$ or $q=2$. First let $q>2$ and assume the condition in Theorem 1(i).

Proof of Theorem 1(i): From the above estimate $(*)$ for $i=k \geq 1$, we have

$$
\left|\mathcal{R}_{k}\right| \geq \theta(d)-\alpha(q)-2-\sum_{j=0}^{k-1} \theta\left(d-l_{j}\right)+k(k+1) / 2 .
$$

The right hand side of this inequality is at least

$$
\theta(d)-\alpha(q)-2-\sum_{l=1}^{d-1} \theta(l)+1
$$

because $k(k+1) / 2 \geq 1$ and the sequence $\left(l_{0}, l_{1}, \ldots, l_{k-1}\right)$ is a subsequence of $(d-1, d-$ $2, \ldots, 1)$. Now it follows from Lemma 2 that the above value coincides with $\theta(d-1)+1$. Thus we have $\left|\mathcal{R}_{k}\right| \geq \theta(d-1)+1$.

As the subspace $U_{k}$ of $P G(n, q)$ contains all members of $\mathcal{R}_{k}$, it follows from the above estimate for $\left|\mathcal{R}_{k}\right|$ and Lemma 3 that $U_{k}=P G(n, q)$. Then $n=\operatorname{dim}\left(U_{k}\right)=2 d+\sum_{j=0}^{k-1} l_{j}$. As $\left(l_{0}, l_{1}, \ldots, l_{k-1}\right)$ is a subsequence of $(d-1, d-2, \ldots, 1)$, we conclude that

$$
n=2 d+\sum_{j=0}^{k-1} l_{j} \leq 2 d+\sum_{l=1}^{d-1} l=2 d+d(d-1) / 2=d(d+3) / 2 .
$$

Proof of Theorem 1(ii): In the remainder of this section, we assume that $q=2$ and $\mathcal{A}$ is a $d$-dimensional dual hyperoval in $P G(n, 2)$. To stress on the fact that $\mathcal{A}$ is a dual hyperoval, we use the letter $\mathcal{H}$ instead of $\mathcal{A}$. Then $|\mathcal{H}|=\theta(d)+1$ and the previous estimate $(*)$ for $\left|\mathcal{R}_{i}\right|$ can be read as follows:

$$
(* *) \quad\left|\mathcal{R}_{i}\right| \geq \theta(d)-\sum_{j=0}^{i-1} \theta\left(d-l_{j}\right)+\left(i^{2}+i\right) / 2-1 .
$$

In order to prove that $n \leq D+2, D=d(d+3) / 2$, we separate the proof into several steps.

Step 1. If $l_{k-1} \geq 2$, then $n<d(d+3) / 2$. 
Proof: By assumption, $\left(d+1-l_{0}, \ldots, d+1-l_{k-1}\right)$ is a subsequence of $(2,3, \ldots$, $d-1)$. Thus it follows from the above estimate ( $* *)$ for $i=k$ and $q=2$ that

$$
\begin{aligned}
\left|\mathcal{R}_{k}\right| & \geq 2^{d+1}-\left(2^{2}+\cdots+2^{d-1}\right)+d+\left(k^{2}+k-4\right) / 2 \\
& =2^{d+1}-\left(2^{d}-1-3\right)+d+\left(k^{2}+k-4\right) / 2 \\
& =2^{d}+d+\left(k^{2}+k+4\right) / 2>2^{d}=\theta(d-1)+1 .
\end{aligned}
$$

As $U_{k}$ contains all members of $\mathcal{R}_{k}$, this together with Lemma 3 implies that $U_{k}=P G(n, q)$. Hence $n=\operatorname{dim}\left(U_{k}\right)=2 d+\left(l_{0}+\cdots+l_{k-1}\right) \leq 2 d+((d-1)+\cdots+2)<d(d+3) / 2$.

Thus in the following, we assume that $l_{k-1}=1$.

Step 2. If $k=1$ and $l_{k-1}=1$, then $n \leq d(d+3) / 2$. Equality holds if and only if $d=2$.

Proof: $\quad$ Suppose $k=1$. Then $l_{0}=l_{k-1}=1$ by our assumption, and $\operatorname{dim}\left(U_{0} \cap X\right) \geq d-$ $l_{0}=d-1$ for every member $X$ of $\mathcal{H}$ by the definition of $l_{0}$. Then $n \leq \operatorname{dim}\left(U_{0}\right)+1=2 d+1$ by Lemma 4 . As $2 d+1 \leq d(d+3) / 2$ for $d \geq 2$ (the equality holds iff $d=2$ ), the claim follows.

Hence in the following, we assume that $l_{k-1}=1$ and $k \geq 2$. In particular, the notation $\mathcal{R}_{k-2}$, $U_{k-2}$, and $l_{k-2}$ make sense. Furthermore, as $\left(l_{0}, \ldots, l_{k-3}\right)$ with $k \geq 3$ (resp. $\left(l_{0}, \ldots, l_{k-2}\right)$ with $k \geq 2$ and $\left(l_{0}, \ldots, l_{k-1}\right)$ with $\left.k \geq 1\right)$ is a subsequence of $(d-1, \ldots, 3)$ (resp. $(d-1, \ldots, 2)$ and $(d-1, \ldots, 1))$, the previous estimate $(* *)$ for $\left|\mathcal{R}_{i}\right|$ to $q=2$ and $i=k-2$ shows that:

$$
\begin{aligned}
\left|\mathcal{R}_{k-2}\right| & \geq\left(2^{d+1}-1\right)-\left\{\left(2^{(d+1)-(d-1)}-1\right)+\cdots+\left(2^{(d+1)-3}-1\right)\right\}+\left(k^{2}-3 k\right) / 2 \\
& =2^{d+1}-\left(2^{2}+\cdots+2^{d-2}\right)-1+(d-3)+\left(k^{2}-3 k\right) / 2 \\
& =2^{d+1}-\left(2^{d-1}-4\right)+(d-4)+\left(k^{2}-3 k\right) / 2 \\
& =3 \cdot 2^{d-1}+d+\left(k^{2}-3 k\right) / 2
\end{aligned}
$$

for $k \geq 2$ (note that this is trivially true for $k=2$ ), and similarly,

$$
\begin{aligned}
\left|\mathcal{R}_{k-1}\right| & \geq\left(2^{d+1}-1\right)-\left\{\left(2^{(d+1)-(d-1)}-1\right)+\cdots+\left(2^{(d+1)-2}-1\right)\right\}+\left(k^{2}-k-2\right) / 2 \\
& =2^{d+1}-\left(2^{2}+\cdots+2^{d-1}\right)-1+(d-2)+\left(k^{2}-k-2\right) / 2 \\
& =2^{d+1}-\left(2^{d}-4\right)+(d-3)+\left(k^{2}-k-2\right) / 2 \\
& =2^{d}+d+\left(k^{2}-k\right) / 2
\end{aligned}
$$

for $k \geq 1$.

We now divide the cases according as there is a member $Y$ of $\mathcal{R}_{k-2}$ with $\operatorname{dim}\left(Y \cap U_{k-1}\right)<$ $d-1$. 
Step 3. If there is a member $Y$ of $\mathcal{R}_{k-2}$ with $\operatorname{dim}\left(Y \cap U_{k-1}\right)<d-1$, then

$$
n \leq 2 d+\sum_{j=0}^{k-2} l_{j}+l_{k-2}+1
$$

Proof: Assume that a member $Y$ of $\mathcal{R}_{k-2}$ satisfies $\operatorname{dim}\left(Y \cap U_{k-1}\right)=d-l$ for some $l \geq 2$. As $Y \in \mathcal{R}_{k-2}$ and $U_{k-2} \subset U_{k-1}$, we have

$$
d-l=\operatorname{dim}\left(Y \cap U_{k-1}\right) \geq \operatorname{dim}\left(Y \cap U_{k-2}\right) \geq d-l_{k-2},
$$

or equivalently $l_{k-2} \geq l \geq 2$. As $Y \cap U_{k-1}$ contains $\theta(d-l)(\leq \theta(d-2))$ points, there are at least $\left|\mathcal{R}_{k-1}\right|-\theta(d-2)$ members of $\mathcal{R}_{k-1}$ intersecting $Y$ at points outside $U_{k-1}$. As such a member $X\left(\in \mathcal{R}_{k-1}\right)$ intersects $U_{k-1}$ in a subspace of dimension at least $d-1=d-l_{k-1}$, we have

$$
X=\left\langle X \cap U_{k-1}, X \cap Y\right\rangle \subset\left\langle U_{k-1}, Y\right\rangle .
$$

As $\left|\mathcal{R}_{k-1}\right|-\theta(d-2) \geq 2^{d}-\left(2^{d-1}-1\right)>2^{d-1}=\theta(d-2)+1$ by the estimate of $\left|\mathcal{R}_{k-1}\right|$ above, it follows from Lemma 3 that every member of $\mathcal{H}$ intersects the subspace $\left\langle U_{k-1}, Y\right\rangle$ in a subspace of dimension at least $d-1$. Then we have

$$
\begin{aligned}
n & \leq \operatorname{dim}\left(\left\langle U_{k-1}, Y\right\rangle\right)+1=\operatorname{dim}\left(U_{k-1}\right)+l+1 \\
& \leq 2 d+\sum_{j=0}^{k-2} l_{j}+l_{k-2}+1
\end{aligned}
$$

from Lemma 4.

Step 4. If every member $X$ of $\mathcal{R}_{k-2}$ satisfies $\operatorname{dim}\left(X \cap U_{k-1}\right) \geq d-1$, then

$$
n \leq 2 d+\sum_{j=0}^{k-2} l_{j}+2 .
$$

Proof: Suppose every member $X$ of $\mathcal{R}_{k-2}$ is contained in $U_{k-1}$. As $\left|\mathcal{R}_{k-2}\right| \geq 3.2^{d-1}>$ $2^{d}=\theta(d-1)+1$ by the estimate of $\left|\mathcal{R}_{k-2}\right|$ previous to Step 3 , we have $U_{k-1}=P G(n, 2)$ by Lemma 3. Then

$$
\begin{aligned}
n & \leq 2 d+\left(l_{0}+\cdots+l_{k-2}\right) \\
& \leq 2 d+(2+\cdots+(d-1))<d(d+3) / 2 .
\end{aligned}
$$

Thus we may assume that there is a member $Y$ of $\mathcal{R}_{k-2}$ not contained in $U_{k-1}$. By our assumption, this implies that $\operatorname{dim}\left(Y \cap U_{k-1}\right)=d-1$. Then there are at least $\left|\mathcal{R}_{k-2}\right|-$ $\theta(d-1)$ distinct members $X$ of $\mathcal{R}_{k-2}$ with $X \cap Y \notin U_{k-1}$. As $\operatorname{dim}\left(X \cap U_{k-1}\right) \geq d-1$ by our assumption, we have $X=\left\langle X \cap U_{k-1}, X \cap Y\right\rangle \subset\left\langle U_{k-1}, Y\right\rangle$. As $\left.\left|\mathcal{R}_{k-2}\right|-\theta(d-1)\right\rangle$ 
$3.2^{d-1}-2^{d}=2^{d-1}=\theta(d-2)+1$, it follows from Lemma 3 that $\operatorname{dim}\left(\left\langle U_{k-1}, Y\right\rangle \cap X\right) \geq d-1$ for every $X \in \mathcal{H}$. Then it follows from Lemma 4 that

$$
\begin{aligned}
n & \leq \operatorname{dim}\left(\left\langle U_{k-1}, Y\right\rangle\right)+1=\operatorname{dim}\left(U_{k-1}\right)+2 \\
& \leq 2 d+\sum_{j=0}^{k-2} l_{j}+2 .
\end{aligned}
$$

We now complete the proof of Theorem 1(ii). As $l_{k-2} \geq 1+l_{k-1}=2$, we may write $l_{k-2}=2+m$ for some nonnegative integer $m$. As $\left(l_{0}, \ldots, l_{k-2}\right)$ is a subsequence of $(d-1, \ldots, 2+m)$, we have

$$
\begin{aligned}
2 d+\left(l_{0}+\cdots+l_{k-2}\right) & \leq 2 d+((2+m)+\cdots+(d-1)) \\
& =2 d+d(d-1) / 2-(m+1)(m+2) / 2 .
\end{aligned}
$$

Thus if the assumption of Step 3 is satisfied, then

$$
\begin{aligned}
n & \leq d(d+3) / 2-(m+1)(m+2) / 2+(m+2)+1 \\
& =d(d+3) / 2+(m+2)(1-m) / 2+1
\end{aligned}
$$

The last value is less than $d(d+3) / 2$ (resp. is equal to $d(d+3) / 2+1$ and $d(d+3) / 2+2)$, if $m \geq 2$ (resp. $m=1$ and $m=0$ ). Thus the claim follows in this case.

(We may verify the following: if $n=d(d+3) / 2+2$, then $m=0, k=d-1$ and $l_{i}=d-1-i$ for every $i=0, \ldots, d-1$; and if $n=d(d+3) / 2+1$, then $m=1, k=d-2$ and $l_{i}=d-1-i$ for $i=0, \ldots, d-4$ but $l_{d-3}=1$.)

On the other hand, if the assumption of Step 4 is satisfied, then

$$
n \leq d(d+3) / 2-(m+1)(m+2) / 2+2 \text {. }
$$

The right hand side of this inequality is less than $d(d+3) / 2$, if $m \geq 1$, and it is equal to $d(d+3) / 2+1$ if $m=0$.

(If the latter holds, then $k=d-1$ and $l_{i}=d-1-i$ for $i=0, \ldots, d-1$.)

Observing $\left|\mathcal{R}_{k}\right|$, we have the following refinement of Theorem 1(ii) when $d \leq 5$.

Proposition 5 Assume that $\mathcal{H}$ is a d-dimensional dual hyperoval in $P G(n, 2)$. Let $k$ be the integer defined above. If $2 \leq d \leq 5$ or $0 \leq k \leq 2$, then $n \leq d(d+3) / 2$.

Proof: By Step 1 and Step 2 of the proof of Theorem 1(ii), we may assume that $l_{k-1}=1$ and $k \geq 2$. As $k \leq d-1$, this implies that $d \geq 3$.

Assume that $k=2$. Then, from the estimate of $\left|\mathcal{R}_{i}\right|$ with the fact that $l_{1}=1$ and $l_{0} \geq 0$, we have

$$
\left|\mathcal{R}_{2}\right| \geq\left(2^{d+1}-1\right)-\left(\theta\left(d-l_{0}\right)+\theta\left(d-l_{1}\right)\right)+2
$$




$$
\begin{aligned}
& \geq 2^{d+1}-1-\left(2^{d+1-2}+2^{d}-2\right)+2 \\
& =2^{d-1}+3
\end{aligned}
$$

As $U_{2}$ contains all members of $\mathcal{R}_{2}$, it follows from Lemma 3 that $U_{2}=P G(n, q)$. Hence $n \leq \operatorname{dim}\left(U_{2}\right)=2 d+l_{0}+l_{1} \leq 2 d+1+(d-1)=3 d$. As $3 d \leq d(d+3) / 2$ for every $d \geq 3$, we have $n \leq d(d+3) / 2$.

Thus we may assume that $k \geq 3$. As $d-1 \geq k$, we have $d \geq 4$. As $\left(l_{0}, \ldots, l_{k-1}\right)$ is a subsequence of $(d-1, \ldots, 1)$, we have the following estimate of $\left|\mathcal{R}_{k}\right|$ :

$$
\begin{aligned}
\left|\mathcal{R}_{k}\right| & \geq\left(2^{d+1}-1\right)-\left\{\left(2^{2}-1\right)+\cdots+\left(2^{d}-1\right)\right\}+\left(k^{2}+k-2\right) / 2 \\
& =2^{d+1}-\left(2^{d+1}-4\right)+(d-1)-1+\left(k^{2}+k-2\right) / 2 \\
& =d+\left(k^{2}+k+2\right) / 2 .
\end{aligned}
$$

Thus the subspace $U_{k}$ contains at least $\left|\mathcal{R}_{k}\right|+k+2\left(\geq d+\left(k^{2}+3 k+6\right) / 2\right)$ distinct members of $\mathcal{H}$. As $k \geq 3$ and $d \geq 4$, we have $d+\left(k^{2}+3 k+6\right) / 2 \geq d+12 \geq 16>\theta(4)=15$. Then it follows from Lemma 3 that $U_{k}=P G(n, 2)$ if $d=4$ or 5. Then $n=\operatorname{dim}\left(U_{k}\right) \leq$ $2 d+(1+\cdots+(d-1))=d(d+3) / 2$.

\section{Constructions of dual arcs}

\subsection{Veronesean construction}

Let $V$ (resp. $W$ ) be a vector space over $G F(q)$ of rank $d+1$ (resp. $D+1=d+1+\left(\begin{array}{c}d+1 \\ 2\end{array}\right)$, $D=d(d+3) / 2$ ) with basis $\mathbf{e}_{i}, i=0, \ldots, d$ (resp. $\left.\mathbf{e}_{i j}, 0 \leq i \leq j \leq d\right)$. The Veronesean map $\zeta$ is the map from $P G(V) \cong P G(d, q)$ into $P G(W) \cong P G(D, q)$ defined by

$$
\left[\sum_{i=0}^{d} x_{i} \mathbf{e}_{i}\right] \mapsto\left[\sum_{0 \leq i \leq j \leq d} x_{i} x_{j} \mathbf{e}_{i j}\right],
$$

where $[\mathbf{x}]$ is the projective point represented by a vector $\mathbf{x}$. The image of $\zeta$ is called the quadric Veronesean and denoted by $\mathcal{V}_{d}$. See [4, Section 25.1] for fundamental properties of the Veronesean.

We denote by $b$ and $B$ the nondegenerate symmetric bilinear forms on $V$ and $W$ defined respectively by

$$
\begin{aligned}
b\left(\sum_{i=0}^{d} x_{i} \mathbf{e}_{i}, \sum_{i=0}^{d} y_{i} \mathbf{e}_{i}\right) & :=\sum_{i=0}^{d} x_{i} y_{i}, \\
B\left(\sum_{0 \leq i \leq j \leq d} x_{i j} \mathbf{e}_{i j}, \sum_{0 \leq i \leq j \leq d} y_{i j} \mathbf{e}_{i j}\right) & :=\sum_{0 \leq i \leq j \leq d} x_{i j} y_{i j} .
\end{aligned}
$$


For a vector $\mathbf{x} \in V$, we denote by $\mathbf{x}^{\perp}$ the subspace of $V$ orthogonal to $\mathbf{x}$ with respect to $b$ :

$$
\mathbf{x}^{\perp}:=\{\mathbf{y} \in V \mid b(\mathbf{x}, \mathbf{y})=0\} .
$$

For a projective point $P=[\mathbf{x}]$ of $P G(V)$, we now define the subspace $A(P)$ of $P G(W)$ by

$$
A(P):=\left\{\mathbf{z} \in W \mid B(\mathbf{z},(\mathbf{y}) \zeta)=0 \text { for every } \mathbf{y} \in \mathbf{x}^{\perp}\right\}
$$

We give an explicit description of the subspace $A(P)$.

Lemma 6 If $P=[\mathbf{x}]$ with $\mathbf{0} \neq \mathbf{x}=\sum_{i=0}^{d} x_{i} \mathbf{e}_{i} \in V$, then $A(P)$ is a subspace of $W$ of rank $d+1$ with a basis

$$
\mathbf{f}_{j}:=\sum_{i=0}^{d} x_{i} \mathbf{e}_{i j} \text { for } j=0, \ldots, d, \text { where } \mathbf{e}_{i j} \text { denotes } \mathbf{e}_{j i} \text { if } j>i
$$

Proof: Take any vector $\mathbf{y}=\sum_{i=0}^{d} y_{i} \mathbf{e}_{i}$ of $\mathbf{x}^{\perp}$. Then $b(\mathbf{x}, \mathbf{y})=\sum_{i=0}^{d} x_{i} y_{i}=0$ and $(\mathbf{y}) \zeta=$ $\sum_{0 \leq i \leq j \leq d} y_{i} y_{j} \mathbf{e}_{i j}$. As $\mathbf{f}_{j}=x_{0} \mathbf{e}_{0 j}+\cdots+x_{j} \mathbf{e}_{j j}+x_{j+1} \mathbf{e}_{j j+1}+\cdots+x_{d} \mathbf{e}_{j d}$ for every $j=$ $0, \ldots, d$, we have $B\left((\mathbf{y}) \zeta, \mathbf{f}_{j}\right)=\left(\sum_{i=0}^{d} x_{i} y_{i}\right) y_{j}=0$. Thus $\mathbf{f}_{j} \in A(P)$ for every $j=0 \ldots, d$.

To show that the vectors $\mathbf{f}_{j}(j=0 \ldots, d)$ are linearly independent, assume $\sum_{j=0}^{d} \beta_{j} \mathbf{f}_{j}=$ $\mathbf{0}$. In the expression of $\sum_{j=0}^{d} \beta_{j} \mathbf{f}_{j}$ as a linear combination of the basis $\mathbf{e}_{i j}(0 \leq i \leq j \leq d)$, the coefficient of $\mathbf{e}_{i i}$ for $i=0 \ldots, d$ (resp. $\mathbf{e}_{i j}$ for $\left.0 \leq i<j \leq d\right)$ is $\beta_{i} x_{i}$ (resp. $\left.\beta_{i} x_{j}+\beta_{j} x_{i}\right)$. Thus $\beta_{i} x_{i}=0$ for every $i=1, \ldots, d$ and $\beta_{i} x_{j}+\beta_{j} x_{i}=0$ for every $0 \leq i<j \leq d$. As $\mathbf{0} \neq \mathbf{x}$, we may assume $x_{0} \neq 0$, without loss of generality. Then the above equations imply that $\beta_{0}=0$ and $\beta_{j} x_{0}=0$ for every $j=1, \ldots, d$, and hence $\beta_{j}=0$ for all $j=0 \ldots, d$.

Now it remains to show that $A(P)$ is a subspace of $W$ of rank $d+1$. As $P G\left(\mathbf{x}^{\perp}\right) \cong$ $P G(d-1, q)$, its image by the Veronesean map $\zeta$ is isomorphic to $\mathcal{V}_{d-1}$, which spans the subspace of $P G(W)$ of dimension $(d-1)(d+2) / 2$, and hence its orthogonal complement $A(P)$ is a subspace of $P G(W)$ of dimension $d(d+3) / 2-(d-1)(d+2) / 2-1=d$.

Proposition 7 With the notation above, the following hold.

(1) The set $\mathcal{A} \mathcal{V}_{d}:=\{A(P) \mid P \in P G(V)\}$ is a d-dimensional dual arc in $P G(D, q)$, $D=d(d+3) / 2$, of size $\left|\mathcal{A} \mathcal{V}_{d}\right|=\theta_{q}(d)$.

(2) For three distinct members $A(P), A(Q)$ and $A(R)$ of $\mathcal{A} \mathcal{V}_{d}$, we have

$$
\text { either }\langle A(P), A(Q)\rangle \supseteq A(R) \text { or } \operatorname{dim}(\langle A(P), A(Q)\rangle \cap A(R))=1 \text {. }
$$

The former holds if and only if $P, Q$ and $R$ lie on a line of $P G(V)$.

(3) For $P=\left[\sum_{i=0}^{d} x_{i} \mathbf{e}_{i}\right]$, the point

$$
h(P):=\left[\sum_{i=0}^{d} x_{i}^{2} \mathbf{e}_{i i}+2 \sum_{0 \leq i<j \leq d} x_{i} x_{j} \mathbf{e}_{i j}\right]=\left[\sum_{i=0}^{d} x_{i} \mathbf{f}_{i}\right]
$$


is the unique point of the subspace $A(P)$ which cannot be expressed as $A(P) \cap A(Q)$ for any point $Q \in P G(V)$ distinct from $P$.

Proof: From Lemma 6, the $d$-dimensional subspace $A\left(\left[\mathbf{e}_{i}\right]\right)$ contains $\left[\mathbf{e}_{i j}\right]$ for every $j=$ $0, \ldots, d$. Thus the subspace of $P G(W)$ spanned by all $A\left(\left[\mathbf{e}_{i}\right]\right)(i=0, \ldots, d)$ contains a basis of $P G(W)$, and the axiom (DA3) is satisfied.

Now take two distinct points $P$ and $Q$ of $P G(V)$. As the automorphism group of the Veronesean $\mathcal{V}_{d}$ is a subgroup of $\operatorname{Aut}(P G(W))$ induced by $\operatorname{Aut}(P G(V))$ (see [4, 25.1.10]), we may assume that $P=\left[\mathbf{e}_{0}\right]$ and $Q=\left[\mathbf{e}_{1}\right]$. Then Lemma 6 shows that $A(P) \cap A(Q)=$ $\left[\mathbf{e}_{01}\right]$, which verifies the axiom $(D A 1)$. In particular, $\mathcal{A} \mathcal{V}_{d}$ consists of $\theta(d)=|P G(V)|$ members.

Let $R$ be a point of $P G(V)$ distinct from $P$ and $Q$. If $R$ does not lie in the line through $P$ and $Q$, we may assume that $P=\left[\mathbf{e}_{0}\right], Q=\left[\mathbf{e}_{1}\right]$ and $R=\left[\mathbf{e}_{2}\right]$ under the action of $\operatorname{Aut}\left(\mathcal{V}_{d}\right) \cong \operatorname{Aut}(P G(V))$. Then it is immediate to see $A(P) \cap A(Q) \cap A(R)=\emptyset$. Furthermore, $\langle A(P), A(Q)\rangle$ has a basis $\mathbf{e}_{00}, \mathbf{e}_{01}, \mathbf{e}_{11}, \mathbf{e}_{0 i}, \mathbf{e}_{1 i}(i=2, \ldots, d)$, and hence $\langle A(P), A(Q)\rangle \cap A(R)=\left\langle\mathbf{e}_{02}, \mathbf{e}_{12}\right\rangle$.

If $R$ lies on the line through $P=\left[\mathbf{e}_{0}\right]$ and $Q=\left[\mathbf{e}_{1}\right]$, we may assume $R=\left[\mathbf{e}_{0}+\mathbf{e}_{1}\right]$. Then it follows from Lemma 6 that $A(R)$ has a basis $\mathbf{e}_{0 i}+\mathbf{e}_{1 i}(i=0, \ldots, d)$, all vectors of which are contained in $\langle A(P), A(Q)\rangle$. On the other hand, we have $A(P) \cap A(R)=$ $\left[\mathbf{e}_{00}+\mathbf{e}_{01}\right]$ and $A(P) \cap A(Q) \cap A(R)=\emptyset$ in this case as well. Thus the axiom $(D A 2)$ is verified.

The calculations in the above paragraphs show the claim (2).

To check the claim (3), we set $\mathbf{x}:=\sum_{i=0}^{d} x_{i} \mathbf{e}_{i}$ and $h(\mathbf{x}):=\sum_{i=0}^{d} x_{i}^{2} \mathbf{e}_{i i}+2$ $\sum_{0 \leq i<j \leq d} x_{i} x_{j} \mathbf{e}_{i j}$. It is easy to see that $h(\mathbf{x})=\sum_{j=0}^{d} x_{j} \mathbf{f}_{j}$, where $\mathbf{f}_{j}$ is a basis of $A(P)$ given in Lemma 6. In particular, $h(P):=[h(\mathbf{x})] \in A(P)$.

Suppose $h(P)=[h(\mathbf{x})] \in A(Q)$ for some $Q=\left[\mathbf{x}^{\prime}\right]=\left[\sum_{i=0}^{d} x_{i}^{\prime} \mathbf{e}_{i}\right]$ distinct from $P=[\mathbf{x}]$. Then for every $\mathbf{y}=\sum_{i=0}^{d} y_{i} \mathbf{e}_{i}$ with $b\left(\mathbf{x}^{\prime}, \mathbf{y}\right)=0$, the definition of $A(Q)$ implies that $B(h(\mathbf{x}),(\mathbf{y}) \zeta)=0$. Now we have

$$
\begin{aligned}
B(h(\mathbf{x}),(\mathbf{y}) \zeta) & =\sum_{i=0}^{d} x_{i}^{2} y_{i}^{2}+2 \sum_{0 \leq i<j \leq d} x_{i} x_{j} y_{i} y_{j} \\
& =\left(\sum_{i=0}^{d} x_{i} y_{i}\right)\left(\sum_{j=0}^{d} x_{j} y_{j}\right)=\left(\sum_{i=0}^{d} x_{i} y_{i}\right)^{2}
\end{aligned}
$$

Thus $0=\left(\sum_{i=0}^{d} x_{i} y_{i}\right)^{2}$. That is, $b(\mathbf{x}, \mathbf{y})=0$ for all $\mathbf{y} \in\left(\mathbf{x}^{\prime}\right)^{\perp}$. Then the nondegeneracy of $b$ yields $\mathbf{x} \in\left(\left(\mathbf{x}^{\prime}\right)^{\perp}\right)^{\perp}=\left[\mathbf{x}^{\prime}\right]$, which contradicts the hypothesis. Hence there is no point $Q$ of $P G(V)$ other than $P$ with $h(P)=A(P) \cap A(Q)$.

In view of the above explicit model $\mathcal{A} \mathcal{V}_{d}$, the following claim is easy to check.

Corollary 8 The above d-dimensional dual arc $\mathcal{A} \mathcal{V}_{d}$ in $P G(D, q), D=d(d+3) / 2$, of size $\theta(d)$ can be extended to a dual hyperoval if and only if $q$ is even. 
If $q$ is even, the dual hyperoval containing $\mathcal{A} \mathcal{V}_{d}$ is unique, which we denote by $\mathcal{H} \mathcal{V}_{d}$. The additional member $H$ of $\mathcal{H} \mathcal{V}_{d}$ to $\mathcal{A} \mathcal{V}_{d}$ satisfies $\operatorname{dim}(\langle A(P), A(Q)\rangle \cap H)=1$ for any two distinct members $A(P), A(Q)$ of $\mathcal{A} \mathcal{V}_{d}$.

Proof: As $\mathcal{A} \mathcal{V}_{d}$ has the second-maximum size $\theta(d)$, it is extendible to a dual hyperoval if and only if the set $H:=\{h(P) \mid P \in P G(V)\}$ of the "holes" $h(P)$ forms a $d$-dimensional subspace of $P G(W)$ (see also the first paragraph of the proof of Proposition 9). Since $h\left(\left[\mathbf{e}_{i}\right]\right)=\left[\mathbf{e}_{i i}\right]$ for $i=0 \ldots, d$, if $H$ is a subspace, then it coincides with $\left\{\sum_{i=0}^{d} y_{i i} \mathbf{e}_{i i} \mid y_{i i} \in\right.$ $G F(q)\}$. If $q$ is odd, then $h\left(\left[\mathbf{e}_{0}+\mathbf{e}_{1}\right]\right)=\left[\mathbf{e}_{00}+\mathbf{e}_{11}+2 \mathbf{e}_{01}\right]$ does not lie in this subspace. Hence if $q$ is odd, then $\mathcal{A} \mathcal{V}_{d}$ cannot be extended to any dual hyperoval. On the other hand, if $q$ is even, then $h(P)=\left[\sum_{i=0}^{d} x_{i}^{2} \mathbf{e}_{i i}\right]$ for every $P=\left[\sum_{i=0}^{d} x_{i} \mathbf{e}_{i}\right]$ and hence $H$ is in fact a $d$-dimensional subspace of $P G(W)$.

The last statement is easily verified by Lemma 6 .

As for the extendibility to a dual hyperoval, the following result holds in general. (This is a straightforward generalization of [2, Theorem 4.2], which treats $d$-dimensional hyperovals in $P G(2 d, q)$ only.)

Proposition 9 Let $\mathcal{A}$ be an arbitrary d-dimensional dual arc in $P G(n, q)$ of size $|\mathcal{A}|=$ $\theta(d)$. If $q$ is even, then $\mathcal{A}$ can be uniquely extended to a d-dimensional dual hyperoval.

Proof: Let $A$ be any member of $\mathcal{A}$. The map sending each member $X$ of $\mathcal{A}-\{A\}$ to the intersection $X \cap A$ is an injective map from $\mathcal{A}-\{A\}$ into the set $P G(A)$ of projective points of $A$ by $(D A 1)$ and $(D A 2)$. Thus all points of $\mathbf{P}(A)$ except one are realized as the intersections $X \cap A$ for $X \in \mathcal{A}-\{A\}$. Let $h(A)$ (the hole in $A$ ) denote the unique exceptional point on $A$. Then the set $H:=\{h(A) \mid A \in \mathcal{A}\}$ consists of $\theta(d)=|\mathcal{A}|$ points, and $H$ coincides with the set of points $P$ of $P G(n, q)$ with the property that $c(P):=|\{X \in \mathcal{A} \mid P \in X\}|=1$. Thus $\mathcal{A}$ can be completed to a dual hyperoval if and only if $H$ is a subspace, and then $\mathcal{A} \cup\{H\}$ is the only dual hyperoval containing $\mathcal{A}$.

We now show that every $(n-d)$-dimensional subspace $L$ intersects with $H$ nontrivially. Suppose there is a $(n-d)$-dimensional subspace $L$ which does not contain any point of $H$. Then it follows from the first paragraph that $L$ does not contain any point $P$ of $P G(n, q)$ with $c(P)=1$. As $c(P) \leq 2$ for every point $P$ of $P G(n, q)$, then the set $\mathbf{P}(L)$ of points on $L$ is a disjoint union of two subsets $L_{0}$ and $L_{2}$, where

$$
L_{i}:=\{P \in \mathbf{P}(L) \mid c(P)=i\} \quad \text { for } i=0,2 .
$$

We count the following set in two ways:

$$
\{(P, X) \in \mathbf{P}(L) \times \mathcal{A} \mid P \in X\} .
$$

Fixing $P \in \mathbf{P}(L)$ first, the size of this set is calculated to be $0 \cdot\left|L_{0}\right|+2\left|L_{2}\right|$, in particular, it is even. On the other hand, denoting by $d(X)$ the dimension of the intersection $L \cap X$, the size is calculated to be $\sum_{X \in \mathcal{A}} \theta_{q}(d(X))$. Note that as $L$ is of dimension $(n-d)$, we have 
$d(X) \geq 0$. Then, as $q$ is even, we have $\theta_{q}(d(X))=q^{d(X)}+\cdots+q+1 \equiv 1 \quad(\bmod 2)$. Thus the equation $2\left|L_{2}\right|=\sum_{X \in \mathcal{A}} \theta_{q}(d(X))$ implies that

$$
0 \equiv \sum_{X \in \mathcal{A}} 1=|\mathcal{A}|=\theta_{q}(d) \equiv 1 \quad(\bmod 2),
$$

which is a contradiction.

Hence, applying a remarkable characterization of subspaces by Bose and Burton [1], we conclude that $H$ is a subspace of $P G(n, q)$ of dimension $d$. Then it is immediate to see that $\mathcal{A} \cup\{H\}$ is a $d$-dimensional dual hyperoval in $P G(n, q)$.

\subsection{Cap construction and field extension}

Let $V$ be a vector space of rank $d+2$ over $G F(q)$. Assume that $\kappa$ is a cap of $P G(V) \cong$ $P G(d+1, q)$ generating $P G(V)$, that is, $\kappa$ is a subset of projective points of $P G(V)$ such that

(1) for any distinct points $P$ and $Q$ of $\kappa$, there is no point of $\kappa \backslash\{P, Q\}$ on the line of $P G(V)$ through $P$ and $Q$, and

(2) $\kappa$ has $d+2$ linearly independent points.

Consider the exterior square $W:=V \wedge V$ of $V$. It has rank $\left(\begin{array}{c}d+1 \\ 2\end{array}\right)=(d+2)(d+1) / 2$, and hence $P G(W) \cong P G(D, q), D=d(d+3) / 2$. For every point $P=[\mathbf{p}]$ of $\kappa$, we set

$$
X(P):=\{\mathbf{p} \wedge \mathbf{x} \mid \mathbf{x} \in V\} .
$$

Then $X(P)$ does not depend on the choice of a vector $\mathbf{p}$ generating $P$, and is a subspace of $W$ of rank $d+1$, as the map $V \ni \mathbf{x} \mapsto \mathbf{p} \wedge \mathbf{x} \in X(P)$ is a linear surjection with kernel [p].

Proposition 10 For every cap $\kappa$ of $P G(V) \cong P G(d+1, q)$ generating $P G(V)$, the following hold with the notation above.

(i) The family $\mathcal{A}(\kappa):=\{X(P) \mid P \in \kappa\}$ is a d-dimensional dual arc in $P G(W) \cong$ $P G(D, q)$ of size $|\kappa|$.

(ii) The dual arc $\mathcal{A}(\kappa)$ satisfies the following property (Property $(T)$ in $[D F])$ :

$$
\begin{aligned}
& \operatorname{dim}(\langle X(P), X(Q)\rangle \cap X(R))=1 \quad \text { for any mutually distinct three members } \\
& \quad X(P), X(Q), X(R) \text { of } \mathcal{A}(\kappa) .
\end{aligned}
$$

Proof: (i) As we saw above, each member $X(P)$ of $\mathcal{A}(\kappa)$ is a $d$-dimensional subspace of $P G(W)$. By the definition of $X(P)$, it is easy to see that $X(P) \cap X(Q)$ coincides with the point $[\mathbf{p} \wedge \mathbf{q}]$, where $[\mathbf{p}]=P$ and $[\mathbf{q}]=Q$. (This corresponds to the line through $P$ and $Q$ in $P G(V))$. As $\kappa$ is a cap, there is no point on the line through $P$ and $Q$ other than $P$ and $Q$. Thus for a point $R \in \kappa \backslash\{P, Q\}$, the point $X(P) \cap X(R)$, which corresponds to the line of $P G(V)$ through $P$ and $R$, is distinct from $X(P) \cap X(Q)$. Thus $X(P) \cap X(Q) \cap X(R)=\emptyset$. As $\kappa$ 
generates $P G(V)$, it contains points $\left[\mathbf{e}_{i}\right](i=0, \ldots, d+1)$ with $\mathbf{e}_{i}(i=0, \ldots, d+1)$ a basis of $V$. Then the subspace of $W$ spanned by $X\left(\left[\mathbf{e}_{i}\right]\right)$ contains a basis $\mathbf{e}_{i} \wedge \mathbf{e}_{j}(0 \leq i<j \leq d)$ of $W$, and hence $\mathcal{A}(\kappa)$ generates $P G(W)$.

(ii) Let $P=\left[\mathbf{e}_{0}\right], Q=\left[\mathbf{e}_{1}\right], R=\left[\mathbf{e}_{2}\right]$ be three mutually distinct points of $P G(V)$. As $\kappa$ is a cap, $\mathbf{e}_{i}(i=0,1,2)$ can be extended to a basis $\mathbf{e}_{i}(i=0, \ldots, d+1)$ of $V$. Assume that $\mathbf{w}$ is a vector of $W$ lying in both $\langle X(P), X(Q)\rangle$ and $X(R)$. We write $\mathbf{w}=\left(\mathbf{e}_{0} \wedge \mathbf{x}\right)+\left(\mathbf{e}_{1} \wedge \mathbf{y}\right)=\mathbf{e}_{2} \wedge \mathbf{z}$ for $\mathbf{x}=\alpha_{0} \mathbf{e}_{1}+\beta_{0} \mathbf{e}_{2}+\mathbf{x}^{\prime}, \mathbf{y}=\alpha_{1} \mathbf{e}_{0}+\beta_{1} \mathbf{e}_{2}+\mathbf{y}^{\prime}, \mathbf{z}=\alpha_{2} \mathbf{e}_{0}+\beta_{2} \mathbf{e}_{1}+\mathbf{z}^{\prime}$, where $\mathbf{x}^{\prime}, \mathbf{y}^{\prime}$ and $\mathbf{z}^{\prime}$ belong to $\left\langle\mathbf{e}_{i} \mid i=3, \ldots, d+1\right\rangle$ and $\alpha_{i}, \beta_{i} \in G F(q)(i=0,1,2)$. As $\mathbf{e}_{i} \wedge \mathbf{e}_{j}$ $(0 \leq i<j \leq d+1)$ form a basis of $W=V \wedge V$, we find $\mathbf{x}^{\prime}=\mathbf{y}^{\prime}=\mathbf{z}^{\prime}=\mathbf{0}, \alpha_{0}=\alpha_{1}$, $\beta_{0}=-\alpha_{2}, \beta_{1}=-\beta_{2}$. In particular, $\mathbf{w}=\beta_{0}\left(\mathbf{e}_{0} \wedge \mathbf{e}_{2}\right)+\beta_{1}\left(\mathbf{e}_{1} \wedge \mathbf{e}_{2}\right)$, which lies in the line through $X(P) \cap X(R)=\left[\mathbf{e}_{0} \wedge \mathbf{e}_{2}\right]$ and $X(Q) \cap X(R)=\left[\mathbf{e}_{1} \wedge \mathbf{e}_{2}\right]$. Hence we have $\langle X(P), X(Q)\rangle \cap X(R)=\langle X(P) \cap X(R), X(Q) \cap X(R)\rangle$ and the claim follows.

Remark If $\mathcal{A}(\kappa)$ is complete, then $\kappa$ is maximal with respect to inclusion. However, the converse may not hold in general. Many examples of maximal caps are known. With each of them, a dual arc satisfying property $(T)$ can be associated from Proposition 10(ii). It is an interesting problem whether or not it is complete.

Example When $q=2$, the complement $\kappa_{d+1}$ of a hyperplane of $P G(V) \cong P G(d+1,2)$ forms a maximal cap of size $2^{d+1}=\theta_{2}(d)+1$. Hence the resulting $d$-dimensional dual $\operatorname{arc} \mathcal{A}\left(\kappa_{d+1}\right)$ in $P G(D, 2), D=d(d+2) / 2$, is a dual hyperoval. This is nothing more than the Huybrechts dual hyperoval [5] (see also the expositions after [7, Proposition 6.5]). We denote it by $\mathcal{H}\left(\kappa_{d+1}\right)$.

Both $\mathcal{H} \mathcal{V}_{d}$ for $q=2$ (see Corollary 8$)$ and $\mathcal{H}\left(\kappa_{d+1}\right)$ are $d$-dimensional dual hyperovals in $P G(D, 2), D=d(d+3) / 2$. However, the latter satisfies property $(T)$ from Proposition 10 (ii) but the former does not from Proposition 7(2). Thus we obtain:

Proposition 11 Let $q=2$. If $d \geq 2$, there is no automorphism of $P G(d(d+3) / 2,2)$ bijectively sending $\mathcal{H}\left(\kappa_{d+1}\right)$ to $\mathcal{H} \mathcal{V}_{d}$.

Remark Del Fra [3, Theorems 2 and 3] showed that there are two isomorphism classes of 2-dimensional dual hyperovals in $P G(5, q)$ for $q=2$ (note that $D=d(d+3) / 2=5$ for $d=2$ ). For $q=4$, he also showed [3, Theorem 4] that such a dual hyperoval satisfying property $(T)$ is isomorphic to the one with the automorphism group $M_{22}$ acting triply transitively on the $22=\theta_{4}(2)+1$ members. The dual hyperoval $\mathcal{H} \mathcal{V}_{2}$ for $q=4$ belongs to the other class from Proposition 7(2).

As for the field extension, the dual arcs behave nicely. The following is straightforward to verify.

Proposition 12 Let $\mathcal{A}$ be a d-dimensional dual arc in $P G(V) \cong P G(n, q)$, and let $e \geq 1$. For each member $X$ of $\mathcal{A}$, its field extension $X \otimes G F\left(q^{e}\right)$ is a d-dimensional subspace of $P G\left(V \otimes G F\left(q^{e}\right)\right) \cong P G\left(n, q^{e}\right)$, and the family $\mathcal{A} \otimes G F\left(q^{e}\right)$ consisting of $d$ dimensional subspaces $X \otimes G F\left(q^{e}\right)$ for $X \in \mathcal{A}$ is a d-dimensional dual arc in $P G\left(n, q^{e}\right)$ of size $|\mathcal{A}|$. 
Remark It may be an interesting problem to examine when $\mathcal{A} \otimes G F\left(q^{e}\right)$ is complete.

\subsection{Quotients}

For completeness, we give some remark on the dimensions of ambient spaces of the quotients of dual arcs we constructed above. However, we do not attempt to be best possible here. We first recall a definition, which was first given in $[\mathrm{Hu}]$ to dual hyperovals.

Definition Let $\mathcal{A}$ (resp. $\overline{\mathcal{A}}$ ) be a $d$-dimensional dual arc in $P G(U) \cong P G(n, q)$ (resp. $P G(V) \cong P G(m, q)$ with $n \geq m)$. We say that $\mathcal{A}$ covers $\overline{\mathcal{A}}$ (or $\overline{\mathcal{A}}$ is a quotient of $\mathcal{A}$ ), if there exists a linear map $\rho$ from $U$ onto $V$ such that $(X) \rho \in \overline{\mathcal{A}}$ for every member $X$ of $\mathcal{A}$ and that the map $\mathcal{A} \ni X \mapsto(X) \rho \in \overline{\mathcal{A}}$ is a bijection.

Proposition 13 Let $\mathcal{A}$ be a d-dimensional dual arc in $P G(U, q) \cong P G(n, q)$. Then there exists a d-dimensional dual arc $\overline{\mathcal{A}}$ in $P G(m, q)$ with some $2 d \leq m<n$ covered by $\mathcal{A}$ if and only if there is a nonzero subspace $K$ of $U$ of rank $n-m$ such that $\langle X, Y\rangle \cap K=\{\mathbf{0}\}$ for any two distinct members $X, Y$ of $\mathcal{A}$. In particular, if such an arc $\overline{\mathcal{A}}$ exists, then for every integer $l$ with $m \leq l \leq n$, there is a d-dimensional dual arc in $P G(l, q)$ covered by $\mathcal{A}$.

Proof: Assume that there is a $d$-dimensional dual $\operatorname{arc} \overline{\mathcal{A}}$ in $P G(m, q)$ with some $2 d \leq$ $m<n$ covered by $\mathcal{A}$. Let $V$ be the underlying vector space of $P G(m, q)$, and let $\rho$ be a linear surjection from $U$ to $V$ satisfying the conditions in the definition above. We may identify $V$ with the quotient space $U / K$, where $K=\operatorname{Ker}(\rho)$. Then the dual $\operatorname{arc} \overline{\mathcal{A}}$ is given as $\{(X+K) / K \mid X \in \mathcal{A}\}$. As these are subspaces of $U / K$ of rank $d+1$, we should have $X \cap K=\{\mathbf{0}\}$ for every $X \in \mathcal{A}$. Moreover, as $\overline{\mathcal{A}}$ satisfies the axioms (DA1) and (DA2), we have

$$
\begin{aligned}
(X+K) \cap(Y+K) & =(X \cap Y)+K, \text { and }(X+K) \cap(Y+K) \cap(Z+K) \\
& =K \text { for any three distinct members } X, Y, Z \text { of } \mathcal{A} .
\end{aligned}
$$

As $\mathcal{A}$ satisfies the axiom ( $D A 2)$, the latter condition is automatically satisfied if the former holds. Hence the existence of $\overline{\mathcal{A}}$ implies the existence of a nonzero subspace $K$ of $U$ such that

$$
\begin{gathered}
X \cap K=\{\mathbf{0}\} \text { and }(X+K) \cap(Y+K)=(X \cap Y)+K \\
\text { for any two distinct members } X, Y \text { of } \mathcal{A} .
\end{gathered}
$$

Conversely, if there exists such a subspace $K$ of $U$, then $\overline{\mathcal{A}}:=\{(X+K) / K \mid X \in \mathcal{A}\}$ is a family of $d$-dimensional subspaces of $P G(U / K)$ satisfying the axioms $(D A i)(i=1,2,3)$.

We will verify that the above two conditions are equivalent to the condition

$$
\langle X, Y\rangle \cap K=\{\boldsymbol{0}\} \text { for any two distinct members } X, Y \text { of } \mathcal{A} .
$$

Assume first that the previous two conditions are satisfied. Then for any vector $\mathbf{x}+\mathbf{y}=\mathbf{k} \in$ $\langle X, Y\rangle \cap K(\mathbf{x} \in X, \mathbf{y} \in Y)$ we have $\mathbf{x}=-\mathbf{y}+\mathbf{k} \in(X+K) \cap(Y+K)=(X \cap Y)+K$ 
from the second condition, and hence $\mathbf{x}=\mathbf{z}+\mathbf{k}^{\prime}$ for some $\mathbf{k}^{\prime} \in K$, where $[\mathbf{z}]=X \cap Y$. Thus $\mathbf{z}+\mathbf{y}=\mathbf{k}-\mathbf{k}^{\prime} \in Y \cap K=\{\mathbf{0}\}$ from the first condition, and hence $\mathbf{x}+\mathbf{y}=\mathbf{x}-\mathbf{z} \in$ $X \cap K=\{\mathbf{0}\}$.

Conversely, if the above condition is satisfied, then we clearly have $X \cap K=\{\boldsymbol{0}\}$ for every $X \in \mathcal{A}$. For $\mathbf{x}+\mathbf{k}=\mathbf{y}+\mathbf{k}^{\prime} \in(X+K) \cap(Y+K)$ with $\mathbf{x} \in X, \mathbf{y} \in Y$ and $\mathbf{k}, \mathbf{k}^{\prime} \in K$, we have $\mathbf{x}-\mathbf{y}=\mathbf{k}^{\prime}-\mathbf{k} \in\langle X, Y\rangle \cap K=\{\mathbf{0}\}$, and hence $\mathbf{x}=\mathbf{y} \in X \cap Y$, as required. Thus the former part of the claim is established.

The latter remark follows by observing that the condition above is satisfied by any nonzero subspace $L$ of $K$ as well.

Now observe that in the proof of [7, Propositon 6.8] the following fact is shown: the Huybrechts dual hyperoval $\mathcal{H}\left(\kappa_{d+1}\right)$ in $P G(D, 2)$ covers a certain $d$-dimensional dual hyperoval $\mathcal{S}_{m, m}^{d+1}$ in $P G(2 d+1,2)$. This together with the above remark implies:

Theorem 14 Let $d$ be any integer with $d \geq 2$, and $n$ be any integer with $2 d+1 \leq n \leq$ $D=d(d+3) / 2$. Then there exists a d-dimensional dual hyperoval in $P G(n, 2)$, which is a quotient of the Huybrechts dual hyperoval $\mathcal{H}\left(\kappa_{d+1}\right)$.

As for the dual arcs covered by the Veronesean dual arc $\mathcal{A} \mathcal{V}_{d}$, the following is verified, though it would be improved much.

Proposition 15 Let $d \geq 5$ and $l$ be any integer with $4 d-2 \leq l \leq D=d(d+3) / 2$. Then, for every prime power $q$ with $q>2$, there is a d-dimensional dual arc in $P G(l, q)$, which is a quotient of $\mathcal{A} \mathcal{V}_{d}$ (or $\mathcal{H} \mathcal{V}_{d}$ if $q$ is even).

Proof: We use the notation in Section 3.1. We also use the symbol $\left(\begin{array}{c}n \\ m\end{array}\right)_{q}$ to denote the number of subspaces of rank $m$ in a vector space of rank $n$ over $G F(q)$ :

$$
\left(\begin{array}{l}
n \\
m
\end{array}\right)_{q}:=\left(q^{n}-1\right)\left(q^{n-1}-1\right) \cdots\left(q^{n-m+1}-1\right) /(q-1)\left(q^{2}-1\right) \cdots\left(q^{m}-1\right)
$$

From Proposition 7(2), for pairs $\{P, Q\}$ and $\left\{P^{\prime}, Q^{\prime}\right\}$ of distinct points of $P G(V)$, we have $\langle A(P), A(Q)\rangle=\left\langle A\left(P^{\prime}\right), A\left(Q^{\prime}\right)\right\rangle$ if and only if the line through $P$ and $Q$ coincides with that through $P^{\prime}$ and $Q^{\prime}$. Thus the $2 d$-dimensional subspace $A(l):=\langle A(P), A(Q)\rangle$ of $P G(W) \cong P G(D, q)$ does not depend on the pair $\{P, Q\}$ of points on a line $l$. We denote by $\mathcal{L}$ the set of all such subspaces: $\mathcal{L}:=\{A(l) \mid l$ : lines of $P G(V)\}$. Then $|\mathcal{L}|=\left(\begin{array}{c}d+1 \\ 2\end{array}\right)_{q}=\left(q^{d+1}-1\right)\left(q^{d}-1\right) /(q-1)\left(q^{2}-1\right)=\theta(d) \theta(d-1) /(q+1)$.

From Proposition 13, to establish the claim for dual arcs covered by $\mathcal{A} \mathcal{V}_{d}$, it suffices to show the existence of a subspace $K$ of $W$ of rank $D-(4 d-2)$ which satisfies $K \cap A(l)=\{\mathbf{0}\}$ for any $A(l) \in \mathcal{L}$. Suppose not. We derive a contradiction by counting the number of triples $(P, K, A(l))$ of projective points $P$ of $P G(W)$, subspaces $K$ of $P G(W)$ of dimension $m:=$ $D-4 d+1$ and members $A(l)$ of $\mathcal{L}$ with $P \in K \cap A(l)$. Note that $m=d(d-5) / 2+1 \geq 1$ as $d \geq 5$. 
First fix a member $A(l)$ of $\mathcal{L}$. The $2 d$-dimensional subspace $A(l)$ has exactly $\theta(2 d)$ points $P$, each of which is contained in exactly $\left(\begin{array}{c}D \\ m\end{array}\right)_{q}$ subspaces $K$ of $P G(W)$ of dimension $m$. Thus the number of such triples is

$$
|\mathcal{L}| \theta(2 d)\left(\begin{array}{l}
D \\
m
\end{array}\right)_{q}=\theta(d) \theta(d-1) \theta(2 d)\left(\begin{array}{l}
D \\
m
\end{array}\right)_{q} /(q+1) .
$$

On the other hand, it follows from our hypothesis that for every subspace $K$ of $P G(W)$ of dimension $m$, there is at least one member $A(l)$ of $\mathcal{L}$ such that $K \cap A(l)$ contains a point. Thus the above number is at least $\left(\begin{array}{c}D+1 \\ m+1\end{array}\right)_{q}=\left(\begin{array}{c}D \\ m\end{array}\right)_{q}\left(q^{D+1}-1\right) /\left(q^{m+1}-1\right)$, and hence we have

$$
\theta(d) \theta(d-1) \theta(2 d) \theta(m) \geq(q+1) \theta(D) .
$$

Now, as $q>2$, it is easy to see that $\theta(a) \theta(b)<(q /(q-1)) \theta(a+b)$ for every $a, b \geq 1$. Applying this to the left hand side of the inequality above, we get $(q /(q-1)) \theta(2 d+$ $m) \cdot(q /(q-1)) \theta(2 d-1)>(q+1) \theta(D)$, and hence $\left(q^{3} /(q-1)^{3}\right) \theta(4 d+m-1)=$ $\left(q^{3} /(q-1)^{3}\right) \theta(D)>(q+1) \theta(D)$, as $m=D-4 d+1$. This implies that $q^{3}>(q+1)(q-$ $1)^{3}=q^{4}-2 q^{3}+2 q-1$, that is, $q^{3}(q-3)+(2 q-1)<0$ and $q=2$. This is against that $q>2$. (Note that no contradiction is obtained when $q=2$, because in this case the above inequality holds for every $d \geq 5$.) Hence there exists a subspace $K$ of rank $D-(4 d-2)$ with $K \cap A(l)=\{\mathbf{0}\}$ for all members $A(l)$ of $\mathcal{L}$, and the claim on the quotients of $\mathcal{A} \mathcal{V}_{d}$ holds.

To show the claim on quotients of $\mathcal{H} \mathcal{V}_{d}$, assume that $q$ is even with $q>2$. As a quotient of $\mathcal{A} \mathcal{V}_{d}$ in $P G(l, q)$ with $4 d-1 \leq l \leq D$ has $\theta(d)$ members, it follows from Proposition 9 that it can be uniquely extended to a dual hyperoval $\overline{\mathcal{H}}$ in $P G(l, q)$. The additional $d$ dimensional subspace is the set of holes (see the proof of Proposition 9), and hence it bijectively corresponds to the unique member $\{h(P) \mid P \in P G(V)\}$ of $\mathcal{H} \mathcal{V}_{d} \backslash \mathcal{A} \mathcal{V}_{d}$. Thus the dual hyperoval $\overline{\mathcal{H}}$ is covered by $\mathcal{H} \mathcal{V}_{d}$.

\section{Acknowledgment}

Most of the works involved in this paper were done at "Salon de Thé". I express my hearty thanks to each staff member there for allowing me to spend the most happy times while I stayed in Osaka.

\section{References}

1. R.C. Bose and R. Burton, "A characterization of flat spaces in a finite geometry and the uniqueness of the Hamming and the MacDonald codes," J. Combin. Theory 1 (1966), 96-104.

2. B. Cooperstein and J.A. Thas, "On generalized k-arcs in PG(2n, q)," Ann. Combin. 5 (2001), 141-152.

3. A. Del Fra, "On $d$-dimensional dual hyperovals," Geometriae Dedicata 79 (2000), 157-178.

4. J.W.P. Hirschfeld and J.A. Thas, General Galois Geometries, Oxford University Press, 1993.

5. C. Huybrechts, "Dimensional dual hyperovals in projective spaces and c.AG* geometries," Discrete Math. 255 (2002), 193-223. 
6. C. Huybrechts and A. Pasini, "Flag-transitive extensions of dual affine spaces," Contrib. Algebra Geom. 40 (1999), 503-532.

7. A. Pasini and S. Yoshiara, "On a new family of flag-transitive semibiplanes," European J. Combin. 22 (2001), 529-545.

8. A. Pasini and S. Yoshiara, "New distance regular graphs arising from dimensional dual hyperovals," European J. Combin. 22 (2001), 547-560.

9. J.A. Thas and H. van Maldeghem, "Characterizations of the finite quadric Veroneseans $\mathcal{V}_{n}^{2^{n}}$," Quart. J. Math. Oxford Ser. 2.

10. J.A. Thas and H. van Maldeghem, "Characterizations of the finite quadric and Hermitian Veroneseans over finite fields," J. Geom. 76 (2003), 282-293.

11. S. Yoshiara, "A new family of $d$-dimensional dual hyperovals in $P G(2 d+1,2)$," European J. Combin. 20 (1999), 489-503.

12. S. Yoshiara, "On a family of planes of a polar space," European J. Combin. 22 (2001), 107-118. 